\section{Efficacy and improved tolerability of combination therapy with interleukin-1 blockade and MAPK pathway inhibitors for the treatment of Erdheim-Chester disease}

We have read with interest the article by Cohen-Aubart $e t$ al ${ }^{1}$ reporting the efficacy of infliximab in the treatment of ErdheimChester disease (ECD), a rare non-Langerhans histiocytosis. The anti-Tumor Necrosis Factor- $\alpha$ agent demonstrated a variable degree of efficacy, suggesting that infliximab might represent a therapeutic option for moderately severe ECD cases; similar results had been observed with anakinra. ${ }^{2}{ }^{3}$ Nonetheless, following the identification of causative mutations along the mitogen-activated protein kinase(MAPK) pathway, ${ }^{4}$ severe forms of ECD are currently treated with targeted small molecule agents. Specifically, the BRAF inhibitor vemurafenib and the MAPK or extracellular-signal regulated kinase (MEK) inhibitor cobimetinib have been successfully used to treat life-threatening forms of ECD. Although life-saving, these drugs are associated with severe toxicity, which often mandates treatment discontinuation. ${ }^{5}$ Here, we describe the efficacy and improved tolerability of combination therapy with interleukin-1 blockade and targeted MAPK inhibitors in the treatment of ECD.

Among 45 patients with a diagnosis of ECD and followed up at our institution, 25 have been treated with at least one targeted therapy (vemurafenib or cobimetinib). All patients underwent clinical evaluations every 3 months in order to assess treatment efficacy and safety; imaging restaging of disease activity and extension was performed every 6 months. Patient clinical and treatment characteristics are summarised in table 1 . Sixteen patients were treated with vemurafenib, seven with cobimetinib and two were sequentially treated with vemurafenib and cobimetinib, with the former also receiving a second vemurafenib course after the discontinuation of the MEK inhibitor. Cobimetinib and vemurafenib were successful in controlling disease progression and improving clinical manifestations in all patients. However, treatment had to be discontinued in eight cases (29\%); adverse reactions to vemurafenib and cobimetinib were the only cause of treatment discontinuation.

We hypothesised that combination therapy with anakinra might effectively dampen the toxicity related to MAPK pathway inhibition, thus preventing discontinuation of these life-saving drugs. Seven patients from our cohort were treated with a combination therapy including anakinra. In two of these cases, anakinra was initiated following the development of a severe inflammatory adverse event induced by MAPK inhibition. Specifically, one patient with severe ECD sequentially received vemurafenib and cobimetinib; however, treatment with both MAPK inhibitors yielded a severe systemic inflammatory reaction (fever, elevation of acute phase reactants), which mandated discontinuation. Subsequent initiation of anakinra in addition to vemurafenib effectively controlled systemic inflammation. Another patient developed myocarditis while on vemurafenib; he received anakinra as an add-on therapy, which prompted resolution of cardiac inflammation. In both cases, combination therapy with anakinra proved effective and well tolerated. Most importantly, it enabled retention of life-saving treatment regimens with MAPK inhibitors.

A previous study indicated that combination therapy with anakinra and MAPK pathway inhibitors in ECD might result in incremental efficacy. ${ }^{6}$ The present study indicates that anakinra can effectively control and may prevent MAPK inhibitor-related
Table 1 Clinical and treatment characteristics of targeted therapy

courses

\begin{tabular}{lcc|}
\hline & Vemurafenib (n=19) & Cobimetinib (n=9) \\
\hline Age at last follow-up visit, years & $59.3 \pm 12.8$ & $56.2 \pm 12.4$ \\
\hline Male sex (\%) & $17(90)$ & $6(66)$ \\
\hline BRAF V600E mutation (\%) & $19(100)$ & $3(33)$ \\
\hline Clinical manifestations (\%) & & \\
\hline Cardiovascular & $13(68)$ & $56(67)$ \\
\hline Pleuropulmonary & $13(68)$ & $6(67)$ \\
\hline Neurological and/or orbital & $18(95)$ & $9(100)$ \\
\hline Retroperitoneal & $16(84)$ & $6(67)$ \\
\hline Skeletal & $19(100)$ & $9(100)$ \\
\hline Previous therapies (\%) & $16(84)$ & $8(89)$ \\
\hline Targeted therapy & $1(5)$ & $2(22)$ \\
\hline Interferon- $\alpha$-2a & $12(63)$ & $3(33)$ \\
\hline Glucocorticoid & $8(42)$ & $1(11)$ \\
\hline Methotrexate & $3(16)$ & $1(11)$ \\
\hline Tocilizumab & $1(5)$ & $0(0)$ \\
\hline Anakinra & $0(0)$ & $3(33)$ \\
\hline Infliximab & $1(5)$ & $0(0)$ \\
\hline Imatinib & $1(5)$ & $0(0)$ \\
\hline Tofacitinib & $0(0)$ & $1(11)$ \\
\hline Concomitant therapies (\%) & $8(42)$ & $5(56)$ \\
\hline Glucocorticoids & $8(42)$ & $1(11)$ \\
\hline Anakinra & $2(11)$ & $5(56)$ \\
\hline Adverse reactions (\%) & $11(58)$ & $4(44)$ \\
\hline Renal & $6(32)$ & $1(11)$ \\
\hline Cutaneous & $5(26)$ & $3(33)$ \\
\hline Systemic inflammation & $2(11)$ & $1(11)$ \\
\hline Cardiovascular & $3(16)$ & $3(33)$ \\
\hline Haematological & $0(0)$ & \\
\hline Mucosal/gastrointestinal & $0(0)$ & $1(11)$ \\
\hline Hepatic & $1(5)$ & $5(26)$ \\
\hline reatment discontinued (\%) & & \\
\hline
\end{tabular}

adverse events. Thereby, combination therapy with anakinra might prevent discontinuation and increase retention of lifesaving therapies with MAPK inhibitors, especially in the case of inflammation-mediated reactions. Combinatorial approaches might potentially encompass other cytokine-blocking agents, including infliximab. ${ }^{1}$ However, anakinra might represent a particularly suitable add-on therapy, given an excellent record of safety and a short half-life of 6 hours, which allows for prompt treatment discontinuation on resolution of the adverse events.

Corrado Campochiaro $\odot{ }^{1,2}$ Giulio Cavalli, ${ }^{1,2}$ Nicola Farina $\odot, 1,2$ Alessandro Tomelleri $\odot{ }^{1,2}$ Giacomo De Luca, ${ }^{1,2}$ Lorenzo Dagna ${ }^{1,2}$

${ }^{1}$ Unit of Immunology, Rheumatology, Allergy and Rare Diseases, IRCCS San Raffaele Hospital, Milan, Italy

${ }^{2}$ Vita-Salute San Raffaele University, Milan, Italy

Correspondence to Dr Corrado Campochiaro, Unit of Immunology, Rheumatology, Allergy and Rare Diseases, IRCCS San Raffaele Hospital, 20132 Milano, Italy; campochiaro.corrado@hsr.it

Contributors Conception and design: CC, GC, NF. Generation of clinical data: CC GC, AT, GDL, LD. Analysis and interpretation of data: CC, GC, NF. Drafting of the manuscript or revising it critically for important intellectual content: CC, GC, NF, AT. Final approval of the submitted manuscript: CC, GC, NF, AT, GDL, LD.

Funding GC has received funding from AIRC under MFAG 2018-ID 22136 project. Competing interests None declared.

Patient consent for publication Not required.

Provenance and peer review Not commissioned; internally peer reviewed. 
(c) Author(s) (or their employer(s)) 2019. No commercial re-use. See rights and permissions. Published by BMJ.

CC and GC contributed equally.

\section{(A) Check for updates}

To cite Campochiaro C, Cavalli G, Farina N, et al. Ann Rheum Dis Epub ahead of print: [please include Day Month Year]. doi:10.1136/annrheumdis-2019-216610

Received 7 November 2019

Revised 29 November 2019

Accepted 3 December 2019

Ann Rheum Dis 2019;0:1-2. doi:10.1136/annrheumdis-2019-216610

\section{ORCID iDs}

Corrado Campochiaro http://orcid.org/0000-0001-6806-3794

Nicola Farina http://orcid.org/0000-0001-7650-2582
Alessandro Tomelleri http://orcid.org/0000-0002-5440-2078

\section{REFERENCES}

1 Cohen-Aubart F, Maksud P, Emile J-F, et al. Efficacy of infliximab in the treatment of Erdheim-Chester disease. Ann Rheum Dis 2018;77:1387-90.

2 Tomelleri A, Cavalli G, De Luca G, et al. Treating heart inflammation with interleukin-1 blockade in a case of Erdheim-Chester disease. Front Immunol 2018:9:1233.

3 Cohen-Aubart F, Maksud P, Saadoun D, et al. Variability in the efficacy of the IL1 receptor antagonist anakinra for treating Erdheim-Chester disease. Blood 2016;127:1509-12.

4 Cangi MG, Biavasco $\mathrm{R}$, Cavalli $\mathrm{G}$, et al. BRAF ${ }^{\mathrm{V} 600 \mathrm{E}}$-mutation is invariably present and associated to oncogene-induced senescence in Erdheim-Chester disease. Ann Rheum Dis 2015:74:1596-602.

5 Cohen Aubart F, Emile J-F, Carrat F, et al. Targeted therapies in 54 patients with Erdheim-Chester disease, including follow-up after interruption (the love study). Blood 2017:130:1377-80

6 Franconieri F, Martin-Silva N, de Boysson $\mathrm{H}$, et al. Superior efficacy and tolerance of reduced doses of vemurafenib plus anakinra in Erdheim-Chester disease: towards the paradigm of combined targeting and immune therapies. Acta Oncol 2016;55:930-2. 